\title{
THE LACK OF BROAD-LINE REGIONS IN LOW ACCRETION RATE ACTIVE GALACTIC NUCLEI AS EVIDENCE OF THEIR ORIGIN IN THE ACCRETION DISK
}

\author{
Fabrizio Nicastro, ${ }^{1}$ Andrea Martocchia, ${ }^{2,3}$ and Giorgio MatT ${ }^{2}$ \\ Received 2003 February 28; accepted 2003 April 7; published 2003 April 17
}

\begin{abstract}
In this Letter, we present evidence suggesting that the absence or presence of hidden broad-line regions (HBLRs) in Seyfert 2 galaxies is regulated by the rate at which matter accretes onto a central supermassive black hole, in units of the Eddington rate. Evidence is based on data from a subsample of type 2 active galactic nuclei extracted from the Tran spectropolarimetric sample and made up of all those sources that also have good-quality X-ray spectra available and for which a bulge luminosity can be estimated. We use the intrinsic (i.e., unabsorbed) Xray luminosities of these sources and their black hole masses (estimated by using the well-known relationship between nuclear mass and bulge luminosity in galaxies) to derive the nuclear accretion rate in Eddington units. We find that virtually all HBLR sources have accretion rates larger than a threshold value of $\dot{m}_{\text {thres }} \simeq 10^{-3}$ (in Eddington units), while non-HBLR sources lie at $\dot{m} \lesssim \dot{m}_{\text {thres }}$. These data nicely fit predictions from a model proposed by Nicastro in which the broad-line regions (BLRs) are formed by accretion disk instabilities occurring in proximity of the critical radius at which the disk changes from gas pressure dominated to radiation pressure dominated. This radius diminishes with decreasing $\dot{m}$; for low enough accretion rates (and therefore luminosities), the critical radius becomes smaller than the innermost stable orbit and BLRs cannot form.
\end{abstract}

Subject headings: accretion, accretion disks — galaxies: active — quasars: emission lines

\section{INTRODUCTION}

Seyfert 2 galaxies (where only narrow emission lines are visible) are commonly believed to be intrinsically the same as Seyfert 1 galaxies (where both narrow and broad emission lines are visible), the difference being due to orientation. According to the widely accepted unification model for active galactic nuclei (AGNs; Antonucci 1993), type 2 AGNs are seen edgeon, through large columns of circumnuclear obscuring material that prevents the direct view of the nucleus, including the broadline regions (BLRs). This scenario was first proposed by Antonucci \& Miller (1985) to explain the presence of polarized broad lines in the archetypical Seyfert 2 galaxy, NGC 1068, and is now supported by, in addition to spectropolarimetric observations of hidden broad-line regions (HBLRs) in several other sources, X-ray observations, which demonstrate that Seyfert 2 galaxies usually have absorption columns largely exceeding the Galactic ones.

Despite observations that do generally support orientationbased unification models for AGNs, exceptions do exist. Only about $50 \%$ of the brightest Seyfert 2 galaxies show the presence of HBLRs (Tran 2001) in their polarized optical spectra, while the remaining half do not. It has now been convincingly shown that the presence or absence of HBLRs in Seyfert 2 galaxies depends on the AGN luminosity, with the HBLR sources having on average larger luminosities (Lumsden \& Alexander 2001; Gu \& Huang 2002; Martocchia \& Matt 2002; ${ }^{4}$ Tran 2001, 2003). While Lumsden \& Alexander (2001) explained this finding still in the framework of an orientation model, Tran (2001) proposed the existence of a population of galactic nuclei whose activity is powered by starburst rather than accretion onto a

\footnotetext{
${ }^{1}$ Harvard-Smithsonian Center for Astrophysics, 60 Garden Street, Cambridge, MA 02138; fnicastro@cfa.harvard.edu.

${ }^{2}$ Dipartimento di Fisica, Università Roma Tre, via della Vasca Navale 84, I-00146 Rome, Italy; matt@haendel.fis.uniroma3.it.

${ }^{3}$ Observatoire Astronomique, 11 Rue de 1'Université, F-67000 Strasbourg, France; martok@isaac.u-strasbg.fr.

${ }^{4}$ See also http://www.unico.it/ilaria/AGN5/proceedings.html.
}

supermassive black hole and in which, therefore, the BLRs simply do not exist.

In this Letter, we present evidence that suggests that the absence or presence of HBLRs is regulated by the ratio between the X-ray luminosity and the Eddington luminosity, which, in the accretion power scenario, is a measure of the rate at which matter accretes onto the central supermassive black hole. Our explanation is based on the model proposed by Nicastro (2000, hereafter N00), in which the BLRs are formed by accretion disk instabilities occurring in proximity of the critical radius at which the disk changes from gas pressure dominated to radiation pressure dominated. This radius diminishes with decreasing $\dot{m}$; for low enough accretion rates (and therefore luminosities), the critical radius becomes smaller than the innermost stable orbit, and BLRs cannot form. Under the Keplerian assumption, the model naturally predicts that AGNs that are accreting close to the lowest possible $\dot{m}$ must show the broadest possible emission lines in their optical spectra, either hidden (i.e., in polarimetric light), if the nucleus is obscured, or not hidden, if the nucleus is not obscured. An analogous model has been proposed recently by Laor (2003). In both Nicastro's and Laor's models, the existence of BLRs in AGNs is related to the breadth of the broad emission lines (BELs) and based on the observed upper limit of FWHM 25,000 for the BELs. However, while in the N00 model this a consequence of the lines being produced in clouds of gas located at a distance from the source depending on the accretion rate in Eddington units (so the accretion rate is the physical driver of the observed correlation), in Laor's model the driving parameter is the width of the line itself, and no physical origin is proposed (other than the observed correlation between AGN luminosity and line width).

To check this hypothesis and test the above models, we extracted from the Tran (2001) spectropolarimetric sample the subsample of type 2 AGNs that also have good-quality X-ray spectra available (see Martocchia \& Matt 2002). Most of the sources in our sample have [O III] luminosities much lower than $10^{43} \mathrm{ergs} \mathrm{s}^{-1}$. At such relatively low luminosities, stellar 
light from the galaxy may be a strong contaminant in the optical band. X-ray luminosities, instead, are little or not contaminated by stellar components and so are possibly more reliable indicators of the nuclear, accretion-powered activity. For each source of our sample, we then estimate its $2-10 \mathrm{keV}$ intrinsic (i.e., unabsorbed) luminosity and use it as a reliable measure of the nuclear activity. We then estimate the mass of the black hole, using the relation between the mass and the bulge luminosity (Magorrian et al. 1998; Ferrarese \& Merritt 2000). Comparing the black hole mass and the X-ray luminosity, the accretion rate is eventually derived.

The Letter is organized as follows. In $\S 2$, we define and discuss the adopted sample and the methods used to derive the various parameters. In $\S 3$, the results of our analysis are presented, and they are discussed in $\S 4$. Throughout this Letter, a value of $H_{0}=70 \mathrm{~km} \mathrm{~s}^{-1} \mathrm{Mpc}^{-1}$ is adopted.

\section{THE SAMPLE}

We extracted our primary sample from the spectropolarimetric survey of Tran (2001; see also Tran 2003) of all, optically classified, Seyfert 2 galaxies in the CfA and $12 \mu \mathrm{m}$ samples. Optical polarized spectra of the sources in this survey are rather homogeneous in signal-to-noise ratio (Tran 2001), so the lack of polarized broad lines in the optical spectra of about half of the sources in the sample should not be an artifact of dramatic differences in data quality. First we searched for the X-ray properties of the sample (namely, [1] the intrinsic 2-10 keV flux and [2] the equivalent hydrogen column density $N_{\mathrm{H}}$ ) and selected only those sources that had been observed at least once with imaging X-ray satellites. This allowed us to minimize confusion problems, which, at the typical flux level of our sample, may be relevant (Georgantopoulos \& Zezas 2003). In practice, we used data only from ASCA, BeppoSAX, Chandra, and XMM-Newton, either taken from literature, when available and reliable, or (re)analyzed by ourselves. Finally, we applied two further selections, discarding (1) all sources suspected to be Compton-thick and (2) sources known to be strongly variable, in X-rays, on timescales of years (e.g., NGC 7172; Dadina et al. 2001). For Compton-thick sources, no direct measurements of the intrinsic luminosity are available, while highly variable sources cannot be assigned univocally an Xray luminosity. Additional details on our primary sample selection can be found in Martocchia \& Matt (2002). We ended up with a small but reliable sample consisting of 10 HBLR and six non-HBLR sources. Previous works ( $\mathrm{Gu} \&$ Huang 2002; Martocchia \& Matt 2002; Tran 2003) have shown that all HBLR sources have 2-10 keV luminosities larger than $\sim 3 \times 10^{42} \mathrm{ergs} \mathrm{s}^{-1}$, while all non-HBLR sources have luminosities smaller than this value. No correlation with the column density of the absorber is, instead, found. The X-ray luminosity is clearly correlated with the [O III] luminosity (even if with a large scatter), confirming the reliability of the estimates.

For the 16 sources in our primary sample, we then tried to estimate the nuclear accretion rate, in units of Eddington. To calculate $\dot{m}$, we needed to evaluate the mass of the central black hole, which we derived using the Ferrarese \& Merritt (2000) empirical relationship between nuclear mass and bulge luminosity (we used the numerical coefficients they derived from their sample A). The bulge luminosity $L_{\text {bulge }}$ was derived from the empirical correlation between the galaxy's morphological type (i.e., $T$-type) and the bulge-to-total luminosity ratio given by Simien \& de Vaucouleurs (1986)..$^{5}$ The (corrected for extinction) total luminosity and $T$-type were taken from the Third Reference Catalogue of Bright Galaxies (de Vaucouleurs et al. 1991). The complete information was available only for a subset of our primary sample. This further selection reduced then our final primary sample to a total of 10 sources: six HBLRs and four non-HBLRs.

For completeness, and given the limited size of our final primary sample, we also considered a secondary sample, derived by applying the same criteria and rules used to select our primary sample to the $\mathrm{Gu} \&$ Huang (2002) compilation. This compilation contains all Seyfert 2 galaxies with published (between 1995 and 2002) spectropolarimetric information and therefore is not as homogeneous as the Tran (2001) sample in the quality of the polarized optical spectra. This search added three more HBLRs and two more non-HBLRs to our final sample.

For all sources in our final sample, we calculated the accretion rate in Eddington units (actually the ratio between the nuclear bolometric luminosity $L$ and the Eddington luminosity $L_{\mathrm{E}}$ ) assuming a factor of 10 correction between the $2-10 \mathrm{keV}$ and bolometric luminosities (see footnote 6 in $\S 3$ ). Results are summarized in Table 1 (for the sources of our secondary sample, luminosities are slightly different from those reported by Gu \& Huang 2002 because of the different choice of $H_{0}$ ).

\section{RESULTS}

It has been recently shown (Gu \& Huang 2002; Martocchia \& Matt 2002; Tran 2003) that the intrinsic AGN luminosity of the sources of the Tran (2001) sample can be used to clearly separate the two classes of HBLR and non-HBLR sources. Sources with 2-10 keV luminosities larger than the threshold value of $L_{\mathrm{X}}^{\text {thres }} \simeq 3 \times 10^{42} \mathrm{ergs} \mathrm{s}^{-1}$ do show HBLs in their optical polarized spectra, while sources with $L_{\mathrm{X}}<L_{\mathrm{X}}^{\text {thres }}$ do not (see Fig. 1 of Martocchia \& Matt 2002). Here we provide evidence that suggests that this separation is due to differences in nuclear accretion rate, from HBLR to non-HBLR sources.

Figure 1 shows fractional luminosities in units of Eddington luminosities versus black hole masses for all 15 sources of our primary (circles) and secondary (squares) samples. Open symbols in this plot represent HBLR sources, while filled symbols represent non-HBLR sources. We first note that a very broad range of accretion rates is spanned by the sources of our sample (more than 3 orders of magnitude), which are otherwise powered by central black holes with rather homogeneous masses (only a factor of about 15 across the entire sample). Most importantly, Figure 1 clearly shows that HBLR sources are accreting at much faster rates compared to non-HBLR sources. ${ }^{6}$

The threshold value of $\dot{m}_{\text {thres }} \simeq 10^{-3}$ divides up HBLR from non-HBLR sources in the $M_{\mathrm{BH}}-\dot{m}$ plane (Fig. 1, dashed vertical

\footnotetext{
${ }^{5}$ The $M_{\mathrm{BH}^{-}}-\sigma$ correlation is tighter than the $M_{\mathrm{BH}}-L_{\text {bulge }}$ correlation, as discussed in detail by Ferrarese \& Merritt (2000). However, for only four sources (three of them HBLR) in our sample could we find measured nuclear velocity dispersions (only two more can be added, both HBLR, considering all sources in the $\mathrm{Gu} \&$ Huang 2002 compilation). Moreover, sometimes the reported values differ significantly, with dramatic effects on the estimate of the mass, even of an order of magnitude. For these reasons, we were forced to use the $M_{\mathrm{BH}}-L_{\text {bulge }}$ relationship, despite its larger scattering.

${ }^{6}$ We note that this result is independent of the particular value chosen to convert 2-10 keV into bolometric luminosities: a different value would only shift the threshold accretion rate value that separates HBLR from non-HBLR sources. Of course a potentially more serious problem may arise if the sources in our sample have spectral energy distributions that dramatically differ from each other. However, such a random effect would likely destroy rather than create the correlation that we find.
} 
TABLE 1

SOURCE PARAmeters

\begin{tabular}{|c|c|c|c|c|c|}
\hline Source Name & $\begin{array}{c}L_{\mathrm{X}} \\
\left(\times 10^{42} \mathrm{ergs} \mathrm{s}^{-1}\right)\end{array}$ & $\begin{array}{c}M_{\mathrm{bh}} \\
\left(\times 10^{7} M_{\odot}\right)\end{array}$ & $\begin{array}{c}\dot{m} \\
\left(\times 10^{-3} L / L_{\mathrm{E}}\right)\end{array}$ & HBLR & Sample \\
\hline IC 5063 & 8.1 & 28 & 2.2 & Yes & $\mathrm{P}$ \\
\hline Mrk $438 \ldots \ldots$. & 10.5 & 12 & 6.6 & Yes & $\mathrm{P}$ \\
\hline NGC $4388 \ldots \ldots$ & 6.4 & 35 & 1.4 & Yes & $\mathrm{P}$ \\
\hline NGC $5506 \ldots \ldots$ & 8.7 & 9.9 & 6.6 & Yes & $\mathrm{P}$ \\
\hline NGC $6552 \ldots \ldots$ & 3.1 & 15 & 1.5 & Yes & $\mathrm{P}$ \\
\hline Mrk $1210 \ldots \ldots$ & 16.3 & 10 & 13 & Yes & $\mathrm{P}$ \\
\hline NGC $3081 \ldots \ldots$ & 0.67 & 13 & 0.59 & Yes & $\mathrm{S}$ \\
\hline NGC $4507 \ldots \ldots$ & 16.5 & 18 & 8.1 & Yes & $\mathrm{S}$ \\
\hline NGC $5252 \ldots \ldots$ & 11.6 & 36 & 2.8 & Yes & $\mathrm{S}$ \\
\hline M51 ............ & 0.007 & 6.8 & 0.009 & No & $\mathrm{P}$ \\
\hline NGC $3079 \ldots \ldots$ & 0.018 & 1.4 & 0.09 & No & $\mathrm{P}$ \\
\hline NGC $4941 \ldots \ldots$ & 0.086 & 5.1 & 0.13 & No & $\mathrm{P}$ \\
\hline NGC $7582 \ldots \ldots$ & 1.57 & 20 & 0.6 & No & $\mathrm{P}$ \\
\hline NGC $3281 \ldots \ldots$ & 6.28 & 28 & 2.0 & No & $\mathrm{S}$ \\
\hline NGC $7590 \ldots \ldots$ & 0.06 & 6.3 & 0.084 & No & $\mathrm{S}$ \\
\hline
\end{tabular}

Note. - The 2-10 keV X-ray luminosities, black hole masses, and accretion rates (defined as the ratio of bolometric to Eddington luminosity) for our final primary (P) and secondary (S) samples.

line). The only exceptions are NGC 3081 and NGC 3281, both sources from our "secondary" sample. NGC 3081 is an HBLR source, with an accretion rate of $\sim 6 \times 10^{-4}$, a factor of $\sim 2$ below the threshold value, while NGC 3281 is a non-HBLR source accreting at a rate of $\sim 2 \times 10^{-3}$, again a factor of 2 , but above the threshold. We looked at these two exceptions in somewhat greater detail. The flux of NGC 3081 reported in Gu \& Huang (2002) is taken from Maiolino et al. (1998; BeppoSAX data). We reanalyzed those data and found very similar results. However, the spectral parameters are very poorly constrained. If the power-law index is fixed to 2 (instead of the value of 1.7 chosen by Maiolino et al. 1998), an almost equally good fit is found, but the inferred luminosity (and then $\dot{m})$ is twice as large, ${ }^{7}$ moving the source to the right side of our $\dot{m}-M_{\mathrm{BH}}$ plane, and so supporting our main conclusion.

The X-ray luminosity reported by Gu \& Huang (2002) for the second exception in our sample, the non-HBLR source

\footnotetext{
${ }^{7}$ This large uncertainty is due to the low signal-to-noise ratio of the observation coupled with the large value of $N_{\mathrm{H}}$.
}

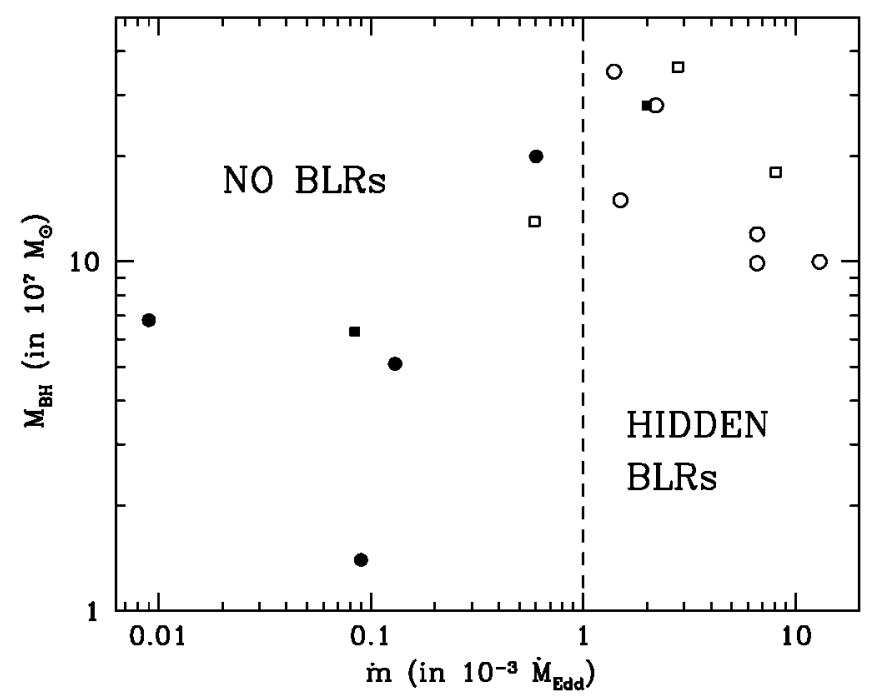

FIG. 1.-Black hole masses vs. accretion rates (defined as $\dot{m}=\dot{M} / \dot{M}_{\mathrm{E}}=$ $\left.L_{\mathrm{bol}} / L_{\mathrm{E}}\right)$. Open and filled symbols refer to HBLRs and non-HBLRs. Circles and squares are sources from our primary and secondary samples, respectively.
NGC 3281, is based on an ASCA observation (Bassani et al. 1999 ) and is underestimated by a factor of $\sim 3$. A subsequent BeppoSAX observation, in fact, demonstrated that the source is actually moderately Compton-thick (Vignali \& Comastri 2002), which implies a higher intrinsic luminosity. This, in turn, implies a higher accretion rate compared to that reported in Table 1 and then amplifies, rather than moderates, the discrepancy found. However, for this source we could not find in the literature details on the spectropolarimetric observation, so it is impossible to judge how significant the upper limit is on the presence of polarized broad lines.

\section{DISCUSSION}

We have presented evidence for a correlation between the presence of HBLs in the polarized optical spectra of nearby Seyfert 2 galaxies and their nuclear accretion rate. Virtually all sources with HBLRs are found above the threshold value of $\dot{m}=10^{-3}$, in the $\dot{m}-M_{\mathrm{BH}}$ plane, and vice versa. Our sample is admittedly small, but both the spectropolarimetric observations (at least for the sources of our primary sample) and the X-ray luminosities are quite reliable. It should be also noted that the many sources of uncertainties in our $\dot{m}$ estimates (namely, [1] the bulge-to-total luminosity ratio vs. T-type, [2] the black hole mass to bulge luminosity correlation, and [3] the bolometric correction) would likely destroy, certainly not artificially create, the observed correlation.

Our findings fit nicely with predictions of the model of N00, in which the BLRs originate from the accretion disk at the transition radius between the gas pressure and radiation pressure dominated regions. This suggests that the accretion rate (N00), rather than the line width (Laor 2003), is indeed the parameter physically responsible for the presence or lack of BLRs in AGNs.

It is remarkable that the threshold value of $\dot{m}_{\text {thres }} \simeq 10^{-3}$ that we find is so close to what is predicted in the framework of the N00 model (i.e., about $4 \times 10^{-3}$ for a black hole mass of $\left.10^{8} M_{\odot}\right)$. The threshold value $\dot{m}_{\text {thres }}$, however, is almost independent of the spin of the black hole, and so, unfortunately, this model cannot be used to discriminate between spinning and nonspinning black holes. This is because in the N00 model the critical radius at which the BLR should form decreases with increasing radiative efficiency in the disk and this decrease is 
almost exactly balanced by the decrease of the radius of the last stable orbit.

Of course, if our explanation is correct, a fraction (set by the relative number of type 1 to type 2 Seyfert galaxies in the nearby universe) of the non-HBLRs should be actually not obscured. In the Gu \& Huang (2002) compilation, four out of the 23 non-HBLR sources with X-ray information available (i.e., 17\%) show little $\left(N_{\mathrm{H}}<10^{21} \mathrm{~cm}^{-2}\right)$ cold X-ray absorption. In the Martocchia \& Matt (2002) sample (i.e., our primary sample, before the bulge luminosity estimate selection), one out of the six non-HBLR sources (again, 17\%) has an estimated column density only a factor of about 3 larger than the Galactic column along that line of sight. In our final sample, only one object (NGC 7590, extracted from the Gu \& Huang 2002 compilation) is virtually unabsorbed. Our estimate of the accretion rate for this object could then in principle be affected by the AGN light in this source, which would artificially increase the total galaxy luminosity (the observed parameter we use), and so the bulge one. However, the $2-10 \mathrm{keV}$ luminosity of this source is very low (see Table 1 ) and so presumably is its $B$ band luminosity. The effect on the estimate of $\dot{m}$, in this case, should therefore be small.

Although suggestive of the existence of a population of unobscured (i.e., type 1) non-HBLR sources, the current statistics is too poor to draw any firm conclusion. Moreover, current estimates of equivalent $\mathrm{H}$ column densities are based on $\mathrm{X}$-ray observations taken with satellites with poor spatial resolutions (mostly ROSAT, ASCA, and BeppoSAX). At such low luminosities, a single spatially unresolved luminous X-ray binary, or a population of those, could lead to an underestimate of the column of matter actually absorbing the nuclear light (Georgantopoulos \& Zezas 2003). Better (i.e., higher spatial resolution and signal-to-noise ratio) and more numerous X-ray data are required to disentangle this issue. Enlarging the sample of low-luminosity supposedly type 2 AGNs with both goodquality optical spectropolarimetric and X-ray (i.e., Chandra and/or XMM-Newton) data would allow us to eventually find a subpopulation of non-HBLR sources, with little or no cold $\mathrm{X}$-ray absorption. These would be the genuine type 1 counterparts of the low accretion rate non-HBLR "true" type 2 sources of our current sample. This population should fall on the left side $\left(\dot{m}<\dot{m}_{\text {thres }}\right)$ of the $\dot{m}-M_{\mathrm{BH}}$ plane (see also Laor 2003).

We note that, recently, few examples of this class of low accretion rate type 1 AGNs may have actually been discovered in X-rays (i.e., Pappa et al. 2001; Georgantopoulos \& Zezas
2003; Boller et al. 2003). The ASCA and Chandra spectra of the optically classified Seyfert 2 galaxy NGC 4698 show little or no absorption (Pappa et al. 2001; Georgantopoulos \& Zezas 2003). The estimated accretion rate for the central AGN in this galaxy is lower than $\dot{m} \lesssim 10^{-4}$ (Georgantopoulos \& Zezas 2003), consistent with our proposed scenario. Similarly, ROSAT and Chandra spectra of 1ES 1927+654 show an unobscured, highly variable steep power-law continuum, typical of narrowline Seyfert 1 galaxies (Boller et al. 2003). This object has been recently reclassified as Seyfert 2 based on its optical spectrum (Bauer et al. 2000); 1ES 1927+654 has an optical ( $B$ band) luminosity of $\sim 10^{43}$ ergs $\mathrm{s}^{-1}$, but no mass estimate is available for its central black hole and thus for the accretion rate in Eddington units. However, given the overall X-ray properties of this object (i.e., steep spectrum and large amplitude variability), we speculate that $1 \mathrm{ES} 1927+654$ is actually accreting at very high rates, in terms of Eddington, and belongs then to the opposite extreme of the line width versus accretion rate correlation in the framework of the N00 model. If this is the case, 1ES $1927+654$ would actually be an optically misidentified narrow-line Seyfert 1 galaxy. The two objects described above have no spectropolarimetric observation, so we do not know whether they have an HBLR or not.

Our findings are based on X-ray luminosities and black hole masses derived from the mass to bulge luminosity relation. It is interesting to note that $\mathrm{Gu} \&$ Huang (2002) also find a similar accretion rate versus HBLR existence correlation (see their Fig. 6), using [O III] (rather than X-ray) luminosities and black hole masses obtained from the $M_{\mathrm{BH}^{-}} \sigma$ correlation.

The results presented here support the N00 model and suggest therefore that the accretion rate is the physical driver of the observed bimodal distribution of HBLR and non-HBLR sources with luminosities. While other explanations (see, e.g., Lumsden \& Alexander 2001; Tran 2001, 2003; Martocchia \& Matt 2002; Gu \& Huang 2002) cannot at present be ruled out, the results presented here are very encouraging and worth checking more thoroughly by enlarging the sample with new spectropolarimetric and/or X-ray observations.

Part of this work was done during a visit of G. M. at CfA, whose hospitality he gratefully acknowledges. We thank Andreas Zezas for enlightening discussions and an anonymous referee for useful comments that helped improved this Letter. We acknowledge financial support from Chandra grant GO23122A (F. N.), MIUR under grant COFIN-00-02-36 (A. M. and G. M.), and CNES (A. M.).

\section{REFERENCES}

Antonucci, R. 1993, ARA\&A, 31, 473

Antonucci, R., \& Miller, J. S. 1985, ApJ, 297, 621

Bassani, L., et al. 1999, ApJS, 121, 473

Bauer, F. E., Condon, J. J., Thuan, T. X., \& Broderick, J. J. 2000, ApJS, 129, 547

Boller, T., et al. 2003, A\&A, 397, 557

Dadina, M., et al. 2001, A\&A, 370, 70

de Vaucouleurs, G., de Vaucouleurs, A., Corwin, H. G., Jr., Buta, R. J., Pasturel, G., \& Fouqué, P. 1991, Third Reference Catalogue of Bright Galaxies (New York: Springer)

Ferrarese, L., \& Merritt, D. 2000, ApJ, 539, L9

Georgantopoulos, J., \& Zezas, A. 2003, ApJL, submitted

Gu, Q., \& Huang, J. 2002, ApJ, 579, 205
Laor, A. 2003, ApJ, in press (astro-ph/0302541)

Lumsden, S. L., \& Alexander, D. M. 2001, MNRAS, 328, L32

Magorrian, J., et al. 1998, AJ, 115, 2285

Maiolino, R., et al. 1998, A\&A, 338, 781

Martocchia, A., \& Matt, G. 2002, preprint (astro-ph/0210332)

Nicastro, F. 2000, ApJ, 530, L65 (N00)

Pappa, A., Georgantopoulos, I., Stewart, G. C., \& Zezas, A. 2001, MNRAS, 326, 995

Simien, F., \& de Vaucouleurs, G. 1986, ApJ, 302, 564

Tran, H. D. 2001, ApJ, 554, L19 2003, ApJ, 583, 632

Vignali, C., \& Comastri, A. 2002, A\&A, 381, 834 